\title{
Megakaryocyte Size and Concentration in the Bone Marrow of Thrombocytopenic and Nonthrombocytopenic Neonates
}

\author{
MARTHA C. SOLA-VISNER, ROBERT D. CHRISTENSEN, ALAN D. HUTSON, AND LISA M. RIMSZA

\begin{abstract}
Department of Pediatrics [M.C.S.-V.], University of Florida, Gainesville, Florida 32611; Division of Neonatology [R.D.C.], Intermountain Health Care, Salt Lake City, Utah 84403; Department of Biostatistics [A.D.H.], University at Buffalo, Buffalo, New York 14214;
\end{abstract} \\ and Department of Pathology [L.M.R.], University of Arizona, Tucson, Arizona 85724
}

\begin{abstract}
Thrombocytopenia is frequent among sick neonates, but little is known about its underlying mechanisms. It is known, however, that neonatal megakaryocytes are smaller and of lower ploidy than their adult counterparts and that smaller megakaryocytes produce fewer platelets than larger, more polyploid, megakaryocytes. We hypothesized that neonatal megakaryocytes would not increase their size in response to thrombocytopenia, thus limiting the ability of neonates to mount a response. To test this, we obtained marrow specimens from thrombocytopenic and nonthrombocytopenic neonates and adults. Megakaryocytes were immunohistochemically stained, quantified using an eyepiece reticle, and measured using an image analysis system with incorporated electronic micrometer. We found that, after adjusting for differences in cellularity, neonates and adults had similar megakaryocyte concentrations. When samples from the same sources were compared (tibial clot and vertebral body sections in neonates, iliac crest biopsies in adults), there were also no differences in megakaryocyte concentration between thrombocytopenic and nonthrombocytopenic subjects. The megakaryocyte diameter, however, was greater in adults than in neonates $(19.4 \pm 3.0$ versus $15.3 \pm 1.7 \mu \mathrm{m}, p<0.0001)$. Thrombocytopenic adults also had a higher proportion of large megakaryocytes than nonthrombocytopenic adults $(p<0.001)$. This was not observed among thrombocytopenic neonates, suggesting a developmental limitation in their ability to increase megakaryocyte size. (Pediatr Res 61: 479-484, 2007)
\end{abstract}

$\mathrm{T}$ hrombocytopenia is a common problem among sick neonates, affecting $20 \%$ to $35 \%$ of neonates admitted to neonatal intensive care units (NICUs) $(1,2)$. In approximately $50 \%$ of cases, the thrombocytopenia is thought to be secondary to increased platelet consumption, mainly associated with sepsis, necrotizing enterocolitis (NEC), or alloantibodies. In the remainder of cases, the mechanism underlying the thrombocytopenia is frequently unclear, although a mounting body of evidence suggests that decreased platelet production causes or complicates many such cases (3-5).

In adults with thrombocytopenia due to increased platelet consumption, the marrow attempts to compensate by increasing megakaryocyte number, size, and ploidy (6-8). It is unknown whether thrombocytopenic neonates can compensate in a similar manner, particularly since megakaryocytes

Received July 6, 2006; accepted November 27, 2006

Correspondence: Lisa Rimsza, M.D., Department of Pathology, University of Arizona, 1501 N. Campbell Ave., P.O. Box 245043, Tucson, AZ 85724; e-mail: rimsza@ ahsc.arizona.edu

Supported by grant HL69990 from the National Institutes of Health (M.S.V., L.R.)

DOI: $10.1203 / p d r .0 b 013 e 3180332 \mathrm{c} 18$ from normal fetuses and neonates are smaller and of lower ploidy than megakaryocytes from adults (9-12). Indeed, the number and size of megakaryocytes in thrombocytopenic neonates have never been systematically evaluated, and it is not known whether neonates are capable of increasing their megakaryocyte size and number in response to platelet consumption.

This study was designed to answer this question by quantifying the megakaryocyte number and size in bone marrow samples from thrombocytopenic and nonthrombocytopenic neonates. Because small megakaryocytes with low DNA content cannot be reliably differentiated from other cell types using hematoxylin and eosin-stained sections alone, we identified the megakaryocytes by applying immunohistochemistry, and then measured their sizes using an image analysis system with an incorporated electronic micrometer. We were then able to calculate the megakaryocyte concentration and average size in each sample, in a manner similar to that described in previous studies in adults $(6,7)$.

\section{PATIENTS AND METHODS}

Study samples. Bone marrow samples from neonates were obtained from two sources. Marrow clot sections were obtained from the tibia of nonthrombocytopenic $(n=9)$ and thrombocytopenic $(n=4)$ living neonates undergoing hematological evaluation, using a technique we described that yields high-quality marrow clots in which the cellularity and morphology are preserved (13). Additionally, vertebral body samples were obtained during postmortem examinations of neonates who died in the NICU at the University of Florida $(n=13)$. Nine of these neonates were thrombocytopenic (platelet count $<100,000 / \mu \mathrm{L}$ ) and four were not thrombocytopenic (platelet count $>100,000 / \mu \mathrm{L})$ at the time of death.

These marrow samples were classified into two groups: (1) thrombocytopenic neonates (four tibial clot sections and nine vertebral body samples) and (2) nonthrombocytopenic neonates (nine tibial clot sections and four vertebral body samples).

To compare the response to thrombocytopenia between neonates and adults, we also assessed bone marrow samples from six nonthrombocytopenic adults undergoing staging of nonhematological malignancies and from eight adult patients with the clinical diagnosis of immune thrombocytopenic purpura (ITP). All these samples were iliac crest core biopsies obtained for clinical purposes. These studies were approved by the Institutional Review Board at the University of Florida. Informed consent was obtained from the parents of the neonates undergoing tibial aspirations. A waiver of informed consent was granted to study the postmortem neonatal and the adult samples, which were all existing at the start of the study and were appropriately deidentified.

Abbreviations: GA, gestational age; ITP, immune thrombocytopenic purpura; NEC, necrotizing enterocolitis; PCA, postconceptional age; Tpo, thrombopoietin 
Immunohistochemistry. Immediately before immunohistochemical staining, the sections were deparaffinized and rehydrated. The slides were then subjected to antigen retrieval in citrate buffer at $80^{\circ} \mathrm{C}$ for $2 \mathrm{~h}$. This lowtemperature technique efficiently restored the immunoreactivity of the CD61 antigen while avoiding the disruption and loss of the fragile bone marrow tissue encountered with the standard high-temperature antigen retrieval protocols. After a 20-min cooling period, the slides were stained on an automated immunostainer (Ventana Nexus, Ventana Medical Systems, Tucson, AZ), using the manufacturer's paraffin protocol. A mouse anti-human CD61 monoclonal antibody (Dako, Denmark) at a concentration of 1:10 was used as the primary antibody. This was followed by the Ventana proprietary mixture of secondary antibodies, streptavidin-horseradish peroxidase, and finally diaminobenzadine as the color substrate. Slides were counterstained with the Ventana's hematoxylin.

CD61-positive cells quantitation. Two of the authors (M.S.V. and L.R.) independently counted the number of $\mathrm{CD}^{+} 1^{+}$cells in each sample, in a blinded fashion, using an eyepiece reticle $\left(250 \mu \mathrm{m}^{2}\right.$ per nine small squares at 400×; Klarman Rulings Inc., Litchfield, NH). Beginning at one end of the section, areas of bone marrow (including fat) were centered in the field, and the number of small squares occupied by marrow were counted, as were the $\mathrm{CD} 1^{+}$cells contained in the squares. Megakaryocyte concentration, calculated as the average of the two independent counts, was then expressed as the number of $\mathrm{CD}^{+} 1^{+}$cells per $250 \mu \mathrm{m}^{2}$ of bone marrow. Trabecular bone spicules, blood vessels, hemodilute, and crushed areas were excluded from counting (Fig. 1). A CD61 ${ }^{+}$cell had to include at least a portion of nucleus to be counted (cytoplasmic fragments were excluded). The percentage of fat was then estimated in each sample by one of the authors (LR), a hematopathologist, according to standard practice. To account for differences in fat content between the samples (particularly between neonates and adults), the megakaryocyte concentration was then adjusted for the percentage of cellularity in each sample and expressed as adjusted megakaryocyte concentration. This was carried out using the following formula:

Adjusted megakaryocyte concentration

$$
=\frac{\text { Measured megakaryocyte concentration } \times 100}{\text { Percent cellularity }}
$$

Megakaryocyte size determination. The diameter of each megakaryocyte in the samples was measured using an automated cellular imaging system that combined color-based imaging with automated microscopy to detect and classify cells of interest (Chromavision, ACIS, San Juan Capistrano, CA).

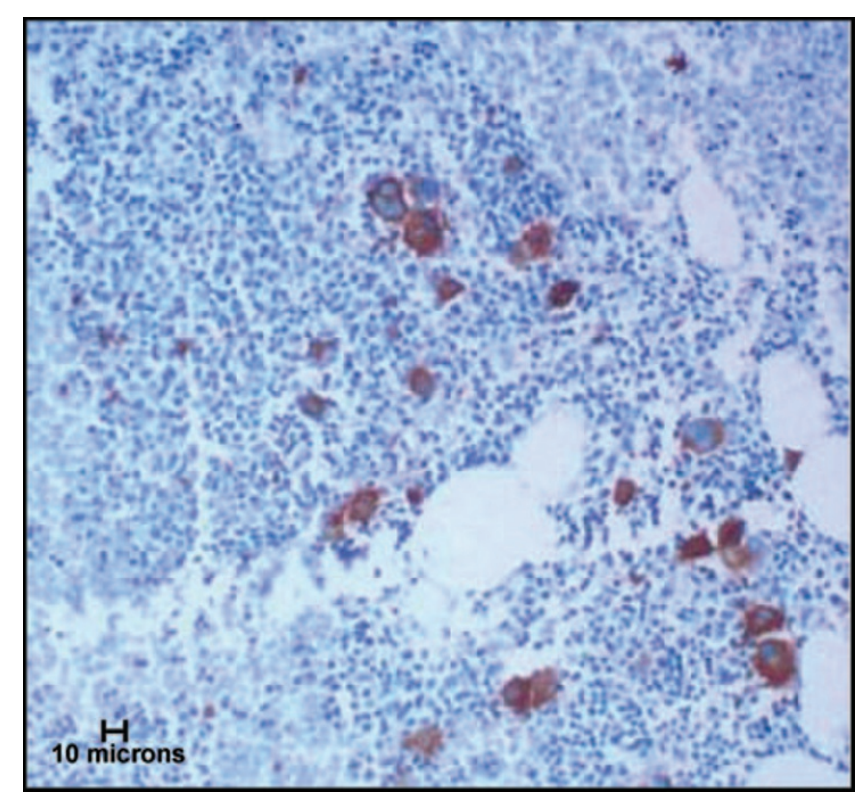

Figure 1. Photomicrograph of a bone marrow clot section from a representative nonthrombocytopenic neonate following immunohistochemical staining with an anti-CD61 antibody. The area of intact bone marrow is in the center of the photomicrograph, and megakaryocytes are identified by their brown cytoplasmic stain. This area of bone marrow is surrounded by a blood clot with only sparse bone marrow elements, which are not considered for the purpose of megakaryocyte quantitation. The marrow cellularity is $<100 \%$.
This system located and captured the images of all $\mathrm{CD} 1^{+}$events in each slide, and then presented them to the user for review. After confirming that the images captured were indeed cells and contained a nucleus, the diameter of each $\mathrm{CD}_{6} 1^{+}$cell was measured with an electronic micrometer and individually recorded.

Statistical methods. Kruskal-Wallis tests were used to compare megakaryocyte concentration, adjusted concentration, and megakaryocyte size between the groups. If the overall $p$ value was found to be significant ( $p$ $<0.05$ ), then Bonferroni's multiple pairwise comparisons were carried out to further examine which specific groups differed from each other. The overall tests were two sided and tested at level alpha $=0.05$. Dot plots were used to graphically compare the four groups for each measure.

Lin's concordance correlation coefficient (14) was used to measure the degree of agreement between the megakaryocyte counts performed independently by the two readers. This coefficient ranges from -1 to 1 , with a value of 1 corresponding to perfect agreement, and 0 corresponding to no agreement.

Individual megakaryocyte diameters were analyzed using a mixed-model analysis of variance with a fixed effect for group and a random effect for subject. The empirical distribution functions for the megakaryocyte diameters for the four groups were graphically generated to examine distributional differences. The $y$ axis represents the megakaryocyte quantiles (the 0.5 quantile is the median), and the $x$ axis the megakaryocyte diameters. All statistical tests were performed using SAS software version 9.

\section{RESULTS}

Study subjects. The demographics and main diagnoses of these infants are displayed in Table 1. As seen, thrombocytopenic and nonthrombocytopenic neonates were similar in terms of gestational age (GA), birth weight, and postconceptional age $[(\mathrm{PCA})=$ gestational age + weeks of life $]$ at the time of bone marrow examination or death. There were also no statistically significant differences in these characteristics between the dead and the alive infants.

Megakaryocyte concentration. When the nonadjusted megakaryocyte concentration (expressed as megakaryocytes/ $250 \mu \mathrm{m}^{2}$ of marrow surface area) was evaluated, adults in general (thrombocytopenic and/or nonthrombocytopenic) had significantly fewer megakaryocytes than neonates in either group ( $p \leq 0.05$; Fig. $2 A$ ). However, we observed no significant differences in megakaryocyte concentration between thrombocytopenic and nonthrombocytopenic neonates (6.0 \pm 4.7 versus $6.8 \pm 2.6$, respectively) or between thrombocytopenic and nonthrombocytopenic adults $(2.4 \pm 0.3$ versus 2.4 \pm 0.4 , respectively). The agreement between the two independent measures of megakaryocyte counts, estimated using Lin's concordance correlation coefficient, was 0.93 , indicating almost perfect agreement between the two sets of counts.

Cellularity and adjusted megakaryocyte concentration. To determine whether the observed differences between adults and neonates were at least partially explained by age-related differences in marrow cellularity, we estimated the cellularity in each sample. All vertebral body samples were $100 \%$ cellular. In contrast, seven of the 13 neonatal tibial clot sections had a cellularity $<100 \%$. As a group, the tibial marrow samples had a mean cellularity of $85 \%$ (range, $50 \%-100 \%$ ). All adult iliac crest biopsy samples exhibited cellularity levels $\leq 60 \%$, with a mean of $40 \%(20 \%-60 \%)$.

After these observations, we adjusted the megakaryocyte concentration in each sample for its cellularity and called this new measure the adjusted megakaryocyte concentration, which constitutes an estimate of megakaryocyte concentration per $250 \mu \mathrm{m}^{2}$ of hematopoietic tissue. Interestingly, we found that the adjusted megakaryocyte concentration was similar in 
Table 1. Characteristics of the four groups of neonates

\begin{tabular}{|c|c|c|c|c|c|c|}
\hline Clinical group & $\begin{array}{l}\text { Source of } \\
\text { sample }\end{array}$ & $n$ & GA (wk) & BW (g) & PCA (wk) & Reasons for BM study or causes of death \\
\hline \multirow[t]{2}{*}{ Thrombocytopenic } & & 13 & $31.4 \pm 5.9$ & $1532 \pm 1240$ & $41.6 \pm 8.4$ & \\
\hline & Tibial & 4 & $34.5 \pm 5.3$ & $1928 \pm 928$ & $44.5 \pm 12.1$ & Congenital HIV (1), liver failure (2), idiopathic (1) \\
\hline \multirow[t]{2}{*}{ Nonthrombocytopenic } & & 13 & $31.5 \pm 4.2$ & $1592 \pm 829$ & $37.9 \pm 4.1$ & \\
\hline & Tibial & 9 & $30.4 \pm 3.5$ & $1352 \pm 540$ & $38.3 \pm 2.6$ & $\begin{array}{l}\text { Idiopathic neutropenia (4), alloimmune neutropenia (2), } \\
\text { autoimmune neutropenia ( } 3 \text { ) }\end{array}$ \\
\hline
\end{tabular}

Results are expressed as mean $\pm 1 \mathrm{SD}$.

$\mathrm{BW}$, birth weight; BM, bone marrow; CDH/ECMO, congenital diaphragmatic hernia/extracorporeal membrane oxygenation; IUGR, intrauterine growth restriction.

nonthrombocytopenic adults and nonthrombocytopenic neonates $(7.8 \pm 2.2$ and $8.4 \pm 3.1$ megakaryocytes, respectively; Fig. $2 B$ ). When the two groups of adults were compared, we also found no difference in adjusted megakaryocyte concentration between the thrombocytopenic and the nonthrombocytopenic group $(6.4 \pm 2.3$ versus $7.8 \pm 2.2$, respectively). In contrast, the thrombocytopenic neonates had a significantly lower adjusted megakaryocyte concentration than their nonthrombocytopenic counterparts $(6.0 \pm 4.7$ versus $8.4 \pm 3.1$, respectively; $p=0.04$ ).

Although not statistically significant, the adjusted megakaryocyte concentration tended to be lower in the vertebral bodies than in the tibial biopsy samples (i.e. $4.4 \pm 3.0$ versus $9.6 \pm 6.1$ for the thrombocytopenic neonates, and
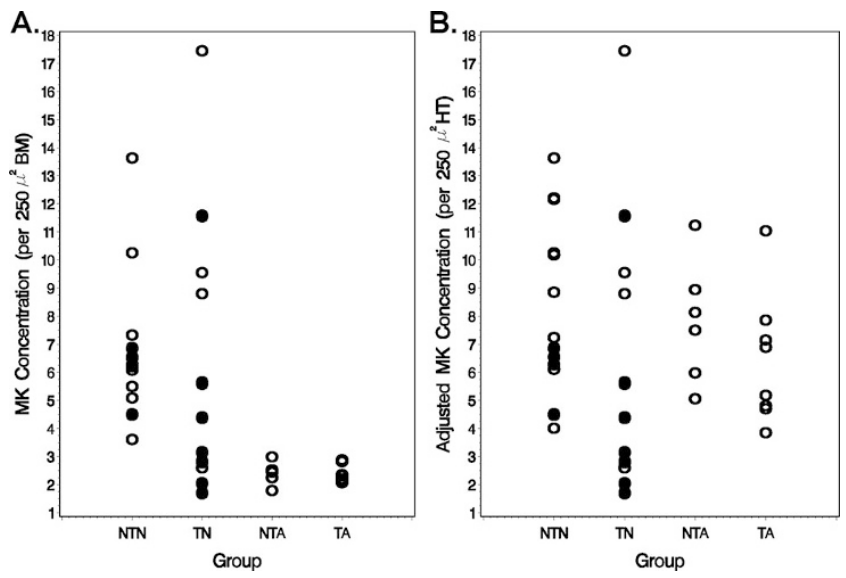

Figure 2. (A) Megakaryocyte concentration (per $250 \mu \mathrm{m}^{2}$ of bone marrow) in bone marrow samples from nonthrombocytopenic neonates (NTN), thrombocytopenic neonates (TN), nonthrombocytopenic adults (NTA), and thrombocytopenic adults (TA). Megakaryocytes were quantified in intact areas of bone marrow, without adjusting for differences in fat content (cellularity). Overall, the adults had significantly lower megakaryocyte concentrations than the neonates. Among the two neonatal groups, vertebral body samples (filled circles) tended to have lower megakaryocyte concentrations than tibial samples (open circles). (B) Adjusted megakaryocyte concentration (per $250 \mu \mathrm{m}^{2}$ of hematopoietic tissue), after correcting for differences in cellularity, in nonthrombocytopenic neonates, thrombocytopenic neonates, nonthrombocytopenic adults, and thrombocytopenic adults. Because the cellularity was lowest in the adult bone marrow biopsy specimens, this adjustment significantly increased the megakaryocyte concentrations in the adult samples, which were then comparable to the neonatal samples. There was also significant variability within the groups, particularly among thrombocytopenic neonates, likely reflecting the different etiologies of the thrombocytopenia and variability related to sample site.
$6.0 \pm 1.1$ versus $9.4 \pm 3.2$ for the nonthrombocytopenic neonates, respectively (Fig. 2B). Because the thrombocytopenic group had comparatively more vertebral body samples ( 9 of 13) than the nonthrombocytopenic group (4 of 13), we hypothesized that the observed differences in adjusted megakaryocyte concentration could be due to differences in the composition of the two groups. To test this, we performed separate analyses for the vertebral body sections (thrombocytopenic versus nonthrombocytopenic) and for the tibial clot sections (thrombocytopenic versus nonthrombocytopenic). These analyses revealed no differences in adjusted megakaryocyte concentration between thrombocytopenic and nonthrombocytopenic neonates when the same source of tissue was used.

Megakaryocyte size. Megakaryocytes from adults (thrombocytopenic and nonthrombocytopenic) were significantly larger than those of thrombocytopenic or nonthrombocytopenic neonates $(p<0.0001)$. However, there were no significant differences in mean megakaryocyte diameter between thrombocytopenic and nonthrombocytopenic neonates (14.5 \pm 1.5 versus $15.3 \pm 1.7 \mu \mathrm{m}$, respectively) or between thrombocytopenic and nonthrombocytopenic adults $(19.5 \pm 2.7$ versus $19.4 \pm 3.0 \mu \mathrm{m}$, respectively). Unlike with the adjusted megakaryocyte concentration, the megakaryocyte diameters were also very similar in vertebral bodies and tibial bone marrow samples.

To investigate whether changes in the megakaryocyte size distribution (such as increases in the proportion of large megakaryocytes in the samples) could exist without translating into changes in the mean diameter, we plotted the diameter of each individual megakaryocyte (total of 5761), classified their relative frequencies according to group, and compared the four groups using an analysis of variance mixed model (Fig. 3). This analysis confirmed that both thrombocytopenic and nonthrombocytopenic neonates had significantly smaller megakaryocytes than both adult groups $(p<0.001)$ and showed no differences in the distribution of megakaryocyte sizes between thrombocytopenic and nonthrombocytopenic neonates. The thrombocytopenic adults, in contrast, had significantly higher proportions of large megakaryocytes than their nonthrombocytopenic counterparts $(p<0.001)$, as evidenced by a shift in their cumulative distribution curve toward larger megakaryocytes (Fig. 3). There was no significant correlation between megakaryocyte number and size in any of the four groups studied. 


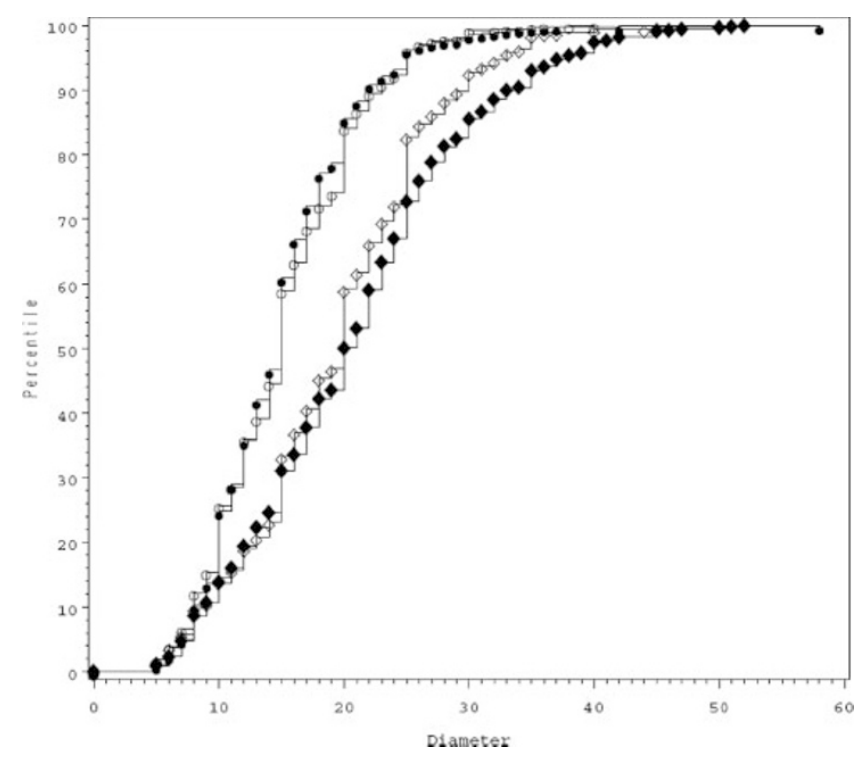

Figure 3. Cumulative distribution plots of megakaryocyte diameters in the bone marrow of thrombocytopenic neonates (filled circles), nonthrombocytopenic neonates (open circles), thrombocytopenic adults (filled diamonds), and nonthrombocytopenic adults (open diamonds). The megakaryocyte diameters are displayed on the $x$ axis and their cumulative distribution on the $y$ axis. Both adult curves are shifted to the right compared with the neonatal curves, indicating the predominance of larger megakaryocytes in these samples. Furthermore, the curve for the thrombocytopenic adults is also shifted to the right compared with that for the nonthrombocytopenic adults, indicating a higher percentage of large megakaryocytes.

\section{DISCUSSION}

Despite the frequency of thrombocytopenia among ill neonates admitted to Neonatal Intensive Care Units, basic questions regarding the underlying mechanisms have remained unanswered. Indeed, little is known about megakaryopoiesis in neonates, partly due to the difficulties in obtaining adequate bone marrow samples from neonates, the rarity of megakaryocytes in the bone marrow (which makes an assessment of their concentration in bone marrow aspirates difficult), and the inability to accurately differentiate small megakaryocytes from cells of other lineages. As a result of these limitations, it is not surprising that studies reporting megakaryocyte concentrations in thrombocytopenic neonates have rendered conflicting results $(1,5,15)$.

In an attempt to overcome these obstacles, we used our published technique for obtaining marrow clot sections in neonates, which contain bone marrow spicules with preserved marrow architecture (13). This method produces extremely small but high-quality specimens and requires no more time or apparent patient discomfort than a traditional marrow aspirate smear preparation. We also obtained vertebral bone marrow sections during postmortem studies in neonates who died with and without thrombocytopenia and applied immunohistochemistry combined with image analysis to obtain an objective measure of megakaryocyte concentration and size in thrombocytopenic and nonthrombocytopenic neonates compared with adults. It has been previously demonstrated that the use of immunohistochemistry improves the detection of megakaryocytes in bone marrow sections (16), a factor that is particularly important when dealing with neonatal samples, in which megakaryocytes are known to be smaller and of lower ploidy and are less conspicuous in routine histologic samples.

Only one previous study evaluated the bone marrow composition of healthy infants at multiple time points $(0-18 \mathrm{mo})$ (17). These investigators obtained bone marrow aspirate smears from neonates and reported that megakaryocytes constituted $0.06 \pm 0.15 \%$ of nucleated cells in those samples. This percentage remained essentially unchanged over the 18 months of the study and is comparable to (or lower than) that usually reported in adult bone marrow aspirates $(<1 \%)(18)$. Our findings using standard clinical counting procedures without adjustment for adipose cell content (cellularity), contradicted the reports by Rosse et al. (17) by showing significantly more megakaryocytes in neonatal marrow tissue or clot sections than in adult bone marrow core biopsy specimens. Three methodological differences between our study and Rosse et al. study probably account for this apparent contradiction: First, we used immunohistochemistry to highlight the megakaryocytes and therefore were less likely to miss the smallest and most immature megakaryocytes (which are more common in neonatal bone marrows). Second, we quantified megakaryocytes in intact marrow spicules or in vertebral body sections, thus minimizing the effects of hemodilution. Third, our measurement represented the megakaryocyte concentration per total marrow surface area, and it is known that cellularity decreases with advancing age $(18,19)$. Indeed, after adjusting for differences in cellularity, we found that nonthrombocytopenic neonates and adults had similar megakaryocyte concentrations per area of hematopoietic tissue. This measure is comparable to expressing megakaryocyte concentration as a percentage of nucleated cells (as would be counted on aspirate smears) and is consistent with the report from Rosse et al. (17). We chose to quantify megakaryocytes per hematopoietic area, as opposed to per nucleated cells, because this allowed us to evaluate the entire sample in each case. However, the issue of peripheral blood hemodilution was avoided by only evaluating areas of intact bone marrow.

It has been common belief that, in the absence of hyporegenerative disorders, neonates have marrows that are 100\% cellular, although a recent study challenged that notion (20). Our finding that the cellularity was invariably $100 \%$ in vertebral bodies, but was lower in several tibial clot sections, highlights the fact that neonatal marrows can have lower cellularities and that some of the reported differences can be related to sampling site.

After correcting for these differences, the concentration of megakaryocytes per area of hematopoietic tissue was significantly lower in thrombocytopenic neonates than in nonthrombocytopenic neonates as a group. When only tibial bone marrow samples obtained from living neonates were analyzed, however, the adjusted megakaryocyte concentration was very similar in thrombocytopenic and nonthrombocytopenic neonates. This suggests that most of the differences seen were among postmortem samples taken from the vertebral bodies of autopsied patients and could therefore have been related to terminal illness leading to suppressed megakaryocytopoiesis. Alternatively, these differences could be explained by the 
presence of a lower megakaryocyte concentration in the vertebral bodies than in the tibia for unknown reasons.

Our finding of a low megakaryocyte size in neonates, compared to adults, is consistent with a large body of literature documenting the smaller size and ploidy of fetal $(9,10,21)$ and cord blood-derived megakaryocytes $(11,12)$. However, this is the first study to directly and objectively measure megakaryocytes in the bone marrow of living neonates. In this regard, it is important to keep in mind that routine tissue processing and paraffin embedding result in significant cell shrinkage $(22,23)$, and therefore our values do not represent true cell diameters. Nevertheless, because all samples were processed and analyzed in similar fashion, our measurements do allow for comparisons between groups. Of interest, we noticed that neonatal megakaryocytes frequently exhibited separation of the nuclear lobes, a finding rare in adult bone marrow and often associated with myelodysplasia. The reason for this difference in nuclear lobation between neonatal and adult megakaryocytes is unknown.

In thrombocytopenic adults, as well as in animal models of adult thrombocytopenia, the megakaryocyte volume increases up to threefold in response to platelet consumption $(6,7)$. This increase in size is usually accompanied by an increase in ploidy $(8,24)$, and it has been recently shown that larger and more polyploid megakaryocytes produce more platelets than do smaller megakaryocytes of lower ploidy (25). In circumstances in which platelets are being rapidly consumed, this increase in megakaryocyte size significantly contributes to expanding the thrombopoietic potential of the marrow. The fact that none of our thrombocytopenic neonates increased the mean megakaryocyte size or the relative proportion of large megakaryocytes (as opposed to thrombocytopenic adults, who significantly increased the proportion of large megakaryocytes in their marrow) suggests that neonates have a limitation in their ability to increase megakaryocyte size and ploidy to compensate for thrombocytopenia.

Typically, adults with ITP have been reported to have increased megakaryocyte number and size in their bone marrows $(7,26)$. However, these features are not present in all cases. Patients with chronic ITP, in particular, often do not have increased megakaryocyte concentrations $(27,28)$ and exhibit decreased concentrations of megakaryocyte colonyforming units (CFU-MK) in megakaryocyte progenitor assays (29). Many of these patients have been shown to have antiplatelet antibodies that also react with megakaryocytes in the bone marrow and can inhibit their proliferation (30). Consistent with these reports, we did not observe an increase in average bone marrow megakaryocyte concentration in our series of ITP patients. Our results are not surprising given the fact that this was an unselected series of ITP patients without reference to clinical scenario. Because bone marrow biopsies are not routinely performed in cases of clear-cut ITP (31), selection bias may have favored obtaining bone marrow biopsies in patients with atypical or chronic courses, explaining the lack of increased megakaryocyte concentration in our small series. Despite these limitations, our data conclusively showed that the proportion of large megakaryocytes signifi- cantly increases in the marrow of adults with thrombocytopenia, whereas neonates do not exhibit this response.

In conclusion, our observations suggest that neonatal megakaryocytes remain small during thrombocytopenia due to different underlying disease processes, a finding that could potentially limit the ability of neonates to increase platelet production in response to increased platelet consumption. However, several potential pitfalls are noted. First, it is possible that our "control" group of nonthrombocytopenic living neonates, many of which were neutropenic at the time of bone marrow examination, also had increased megakaryocytopoiesis as a consequence of increased concentration of cytokines with effects on both neutrophil precursors and megakaryocytes (i.e. granulocyte/macrophage colony-stimulating factor). In this regard, the lack of bone marrow samples from healthy living neonates is a limitation unavoidable in any neonatal human study because ethical considerations absolutely preclude obtaining marrow samples from healthy neonates. Second, it is unclear whether the differences observed between neonates and adults in regard to megakaryocyte size truly represent a developmental limitation in the ability of neonates to increase megakaryocytopoiesis or rather reflect differences in the underlying clinical conditions affecting neonates and adults. In support of the first hypothesis, we recently observed that fetal mice rendered thrombocytopenic through exposure to an antiplatelet antibody (MWReg30, Becton Dickinson) also did not increase their megakaryocyte size and number, whereas adult mice exposed to the same antibody doubled their megakaryocyte mass in the bone marrow and the spleen (Hu $\mathrm{Z}$ et al., Megakaryocyte mass does not increase in response to fetal immune thrombocytopenia. Pediatric Academic Societies' Annual Meeting, April 29-May 2, 2006, San Francisco, CA). These observations in an animal model were consistent with our human observations, thus suggesting that the findings of the present study reflect a true developmental difference between neonates and adults in the ability to respond to thrombocytopenia. The specific mechanisms responsible for this limitation remain to be elucidated, but might be related to our recent observation that neonatal and adult human megakaryocytes have substantial biologic differences in their responses to thrombopoietin (Tpo): although Tpo potently stimulates the maturation of adult megakaryocytes, it inhibits this process in neonatal megakaryocytes. This was demonstrated in our recent work showing that neonatal $\mathrm{CD} 4^{+}$cells cultured in a serum-free system are exquisitely sensitive to Tpo proliferative signals and generate large numbers of megakaryocytes that do not become hyperdiploid, whereas adult $\mathrm{CD} 34^{+}$cells show less proliferation but achieve significantly higher ploidy levels (32). In concordance with these observations, our current study strongly suggests that adult megakaryocytes respond to thrombocytopenia by increasing cell size, whereas neonatal megakaryocytes do not. Furthermore, the few thrombocytopenic neonates in our study who exhibited an increase in megakaryocytopoiesis did so based on increased megakaryocyte number, not size, as would be expected in response to increased Tpo concentrations. The reasons underlying the lack of megakaryocyte proliferation in many of the thrombocytopenic neonates are unknown, but 
likely relate to the presence of undetermined soluble or contact factors in the complex bone marrow hematopoietic microenvironment. Future work will be aimed at better defining the complex in vivo interaction of stimulatory and inhibitory factors that regulate megakaryocytopoiesis during neonatal thrombocytopenia.

Acknowledgments. The authors thank Patty De Haan for technical assistance with the image analysis system and Dr. William Slayton from the Division of Pediatric Hematology/ Oncology at the University of Florida for his critical review of this manuscript.

\section{REFERENCES}

1. Castle V, Andrew M, Kelton J, Giron D, Johnston M, Carter C 1986 Frequency and mechanism of neonatal thrombocytopenia. J Pediatr 108:749-755

2. Mehta P, Vasa R, Neumann L, Karpatkin M 1980 Thrombocytopenia in the high-risk infant. J Pediatr 97:791-794

3. Murray NA, Roberts IA 1996 Circulating megakaryocytes and their progenitors in early thrombocytopenia in preterm neonates. Pediatr Res 40:112-119

4. Murray NA, Watts TL, Roberts IA 1998 Endogenous thrombopoietin levels and effect of recombinant human thrombopoietin on megakaryocyte precursors in term and preterm babies. Pediatr Res 43:148-151

5. Sola MC, Calhoun DA, Hutson AD, Christensen RD 1999 Plasma thrombopoietin concentrations in thrombocytopenic and non-thrombocytopenic patients in a neonatal intensive care unit. Br J Haematol 104:90-92

6. Harker LA 1968 Kinetics of thrombopoiesis. J Clin Invest 47:458-465

7. Harker LA, Finch CA 1969 Thrombokinetics in man. J Clin Invest 48:963-974

8. Tomer A, Friese P, Conklin R, Bales W, Archer L, Harker LA, Burstein SA 1989 Flow cytometric analysis of megakaryocytes from patients with abnormal platelet counts. Blood 74:594-601

9. de Alarcon PA, Graeve JL 1996 Analysis of megakaryocyte ploidy in fetal bone marrow biopsies using a new adaptation of the feulgen technique to measure DNA content and estimate megakaryocyte ploidy from biopsy specimens. Pediatr Res 39:166-170

10. Hegyi E, Nakazawa M, Debili N, Navarro S, Katz A, Breton-Gorius J, Vainchenker W 1991 Developmental changes in human megakaryocyte ploidy. Exp Hematol 19:87-94

11. Levine RF, Olson TA, Shoff PK, Miller MK, Weisman LE 1996 Mature micromegakaryocytes: an unusual developmental pattern in term infants. $\mathrm{Br} \mathrm{J}$ Haematol 94:391-399

12. Olson TA, Levine RF, Mazur EM, Wright DG, Salvado AJ 1992 Megakaryocytes and megakaryocyte progenitors in human cord blood. Am J Pediatr Hematol Oncol 14:241-247

13. Sola MC, Rimsza LM, Christensen RD 1999 A bone marrow biopsy technique suitable for use in neonates. Br J Haematol 107:458-460
14. Lin LI 1989 A concordance correlation coefficient to evaluate reproducibility. Biometrics 45:255-268

15. Andrew M, Castle V, Saigal S, Carter C, Kelton JG 1987 Clinical impact of neonatal thrombocytopenia. J Pediatr 110:457-464

16. Marisavljevic D, Radosevic-Radojkovic N, Rolovic Z 1997 Comparative morphometric study of immunohistochemical versus conventional staining for the evaluation of megakaryocytopoiesis in normal and pathological bone marrow biopsies. Pathol Int 47:301-307

17. Rosse C, Kraemer MJ, Dillon TL, McFarland R, Smith NJ 1977 Bone marrow cell populations of normal infants; the predominance of lymphocytes. J Lab Clin Med $89: 1225-1240$

18. Foucar K. 2001 Bone Marrow Pathology. ASCP Press, Chicago, 175

19. Marley SB, Lewis JL, Davidson RJ, Roberts IA, Dokal I, Goldman JM, Gordon MY 1999 Evidence for a continuous decline in haemopoietic cell function from birth: application to evaluating bone marrow failure in children. Br J Haematol 106:162166

20. Friebert SE, Shepardson LB, Shurin SB, Rosenthal GE, Rosenthal NS 1998 Pediatric bone marrow cellularity: are we expecting too much? J Pediatr Hematol Oncol 20:439-443

21. Ma DC, Sun YH, Chang KZ, Zuo W 1996 Developmental change of megakaryocyte maturation and DNA ploidy in human fetus. Eur J Haematol 57:121-127

22. De Haan BJ, van Goor H, De Vos P 2002 Processing of immunoisolated pancreatic islets: implications for histological analyses of hydrated tissue. Biotechniques 32:612-614, 616, 618-619

23. Gonzalez Santander R, Martinez Cuadrado G, Gonzalez-Santander Martinez M, Monteagudo M, Martinez Alonso FJ, Toledo Lobo MV 1997 The use of different fixatives and hydrophilic embedding media (Historesin and Unicryl) for the study of embryonic tissues. Microsc Res Tech 36:151-158

24. Mazur EM, Lindquist DL, de Alarcon PA, Cohen JL 1988 Evaluation of bone marrow megakaryocyte ploidy distributions in persons with normal and abnormal platelet counts. J Lab Clin Med 111:194-202

25. Mattia G, Vulcano F, Milazzo L, Barca A, Macioce G, Giampaolo A, Hassan HJ 2002 Different ploidy levels of megakaryocytes generated from peripheral or cord blood CD34+ cells are correlated with different levels of platelet release. Blood 99:888-897

26. Abgrall JF, el-Kassar N, Berthou C, Renard I, Cauvin JM, Le Pailleur A, Autrand C, Sensebe L, Guern G, Zilliken P, et al 1992 In vitro megakaryocyte colony formation in patients with idiopathic thrombocytopenic purpura: differences between acute and chronic ITP. Int J Cell Cloning 10:28-32

27. Ohshima K, Kikuchi M, Takeshita M 1995 A megakaryocyte analysis of the bone marrow in patients with myelodysplastic syndrome, myeloproliferative disorder and allied disorders. J Pathol 177:181-189

28. Suvajdzic N, Rolovic Z, Elezovic I, Colovic M 1999 Megakaryocytopoiesis in refractory chronic immune thrombocytopenia. Hematol Cell Ther 41:163-168

29. Abgrall JF, Berthou C, Sensebe L, Le Niger C, Escoffre M 1993 Decreased in vitro megakaryocyte colony formation in chronic idiopathic thrombocytopenic purpura. Br J Haematol 85:803-804

30. McMillan R, Wang L, Tomer A, Nichol J, Pistillo J 2004 Suppression of in vitro megakaryocyte production by antiplatelet autoantibodies from adult patients with chronic ITP. Blood 103:1364-1369

31. Westerman DA, Grigg AP 1999 The diagnosis of idiopathic thrombocytopenic purpura in adults: does bone marrow biopsy have a place? Med J Aust 170:216-217

32. Pastos KM, Slayton W, Rimsza LM, Young L, Sola-Visner MC 2006 Differential effects of recombinant thrombopoietin and bone marrow stromal conditioned media on neonatal vs. adult megakaryocytes. Blood 108:3360-3362 